\title{
Finger Tapping Measures for Parkinson's Disease: Preliminary Evaluation of an Android Application for Data Collection in Australia
}

\author{
Hanna SUOMINEN",a,b,1, Mehika MANOCHA ${ }^{\mathrm{a}}$, Jane DESBOROUGH ${ }^{\mathrm{a}}$, \\ Anne PARKINSON ${ }^{\mathrm{a}}$ and Deborah APTHORP ${ }^{\mathrm{d}}$ \\ ${ }^{\mathrm{a}}$ The Australian National University (ANU), Australia \\ ${ }^{\mathrm{b}}$ Data61/Commonwealth Scientific and Industrial Research Organisation, Australia \\ ${ }^{\mathrm{c}}$ University of Turku, Finland \\ ${ }^{\mathrm{d}}$ University of New England, Australia
}

\begin{abstract}
Parkinson's Disease (PD) is a progressive chronic disorder with a high misdiagnosis rate. Because finger-tapping tasks correlate with its fine-motor symptoms, they could be used to help diagnose and assess PD. We first designed and developed an Android application to perform finger-tapping tasks without trained supervision, which is not always feasible for patients. Then, we conducted a preliminary user evaluation in Australia with six patients clinically diagnosed with PD and sixteen controls without PD. The application could be used in research and healthcare for regular symptom and progression assessment and feedback.
\end{abstract}

Keywords. Electronic health records, evaluation studies, finger tapping, mobile devices, Parkinson Disease, user-computer interface

\section{Introduction}

Parkinson's Disease (PD) is an irreversible, chronic, and progressive disorder, estimated to affect about ten million people worldwide [1]. After PD symptoms emerge, they begin to affect patients' movement and feelings of motivation and reward [2,3]. Motor symptoms of PD include tremor or shaking, which usually begins in one arm or hand, muscle rigidity or stiffness, and slowness, also referred to as bradykinesia [4]. However, signs and symptoms of PD are unique to each person and the treatment should, ideally, be tailored to each person's individual needs [5]. A recent survey of 492 people with PD found most believed self-monitoring would improve their understanding of PD, wellbeing, ability to cope, and communication with their health-care team [6].

The overlap of PD symptoms with other neurological disorders leads to difficulties, delays, and errors in diagnosis, negatively impacting patients [7,8]. A recent study of 1775 patients' experiences of PD diagnosis identified the importance of early diagnosis

${ }^{1}$ Corresponding Author, Hanna Suominen, ANU School of Computing, 145 Science Road, Canberra, ACT 2600, Australia; E-mail: hanna.suominen@anu.edu.au. 
and found misdiagnosis occurred at first consultation for $15 \%$ of participants [9]. This fallibility of clinical diagnosis is recognized and requires periodic diagnosis reviews [5].

Tracking performance in finger-tapping tasks over time provides an alternative or additional measure to gold standard tests for diagnosing PD and tracking its progression. These tests are at present dependent on trained specialists' time to assess each subject's performance in motor (and non-motor) tasks $[4,10,11,12]$. Clinical finger-tapping tasks commonly involve using two fingers of the dominant hand to alternately tap two stationary points for about 30 seconds as a quantitative assessment of bradykinesia, because people with PD tend to have slower, less rhythmic finger taps than controls [13]. In other words, finger tapping performance correlates with the fine motor symptoms of PD [4].

This study reports a preliminary evaluation of an Android application designed to track the progression of PD and inform medical management. The application was developed to record data from repetitive finger-tapping tasks. The underlying research hypothesis was that frequently assessing activities like finger tapping — using a usercentered smartphone-based tool for data collection - could contribute to the body of knowledge about PD and benefit people with PD.

\section{Methods}

The evaluation was methodologically founded on design theories [14,15], which target technology users' requirements in a principled way from day one. It was implemented in cycles of rapid prototyping with $\alpha / \beta$ testing [16] to improve users' experience of the application, with ease of understanding and use as our main design principles [17].

Participants were recruited using social media, flyers, and email. Anyone above the age of 18 years residing in Australia and regularly using a smartphone was eligible to participate. Each participant was interviewed individually and asked to perform a 30second finger-tapping task three times. Interaction involved tapping two circles on the screen with the first two fingers of the dominant hand. Participants completed a questionnaire about the App, which included rating its user interface in terms of simplicity and responsiveness. They were also invited to provide narrative feedback via audio-recorded interviews.

Ethical approval (Protocol 2017/940) was obtained from the ANU Human Research Ethics Committee. Each study participant provided written informed consent.

\section{Results}

Twenty-two people participated in the study (16 controls without PD and 6 people with PD). Their average age was 44 and gender distribution balanced. Most people with PD reported multiple medications. All but one ambidextrous participant were right-handed.

Participants with PD tapped more outside the circles than those in the control group (Table 1, Figure 1). Typically, the left circle had more taps than the right, and most participants began tapping from the left circle. The maximum number of outside taps was recorded as 12 (by a 82-year-old male participant with PD) while the minimum number of outside taps was 0 (by most of the controls). 
Table 1. Comprarson of taps between the controls and people with Parkinson's Disease using a range of measures of total taps.

\begin{tabular}{lrr}
\hline & Controls & People with Parkinson's Disease \\
\hline Average (Standard Deviation) & $114.8(80.4)$ & $60.0(27.5)$ \\
Minimum (Maximum) & $21(85)$ & $41(242)$ \\
\hline
\end{tabular}

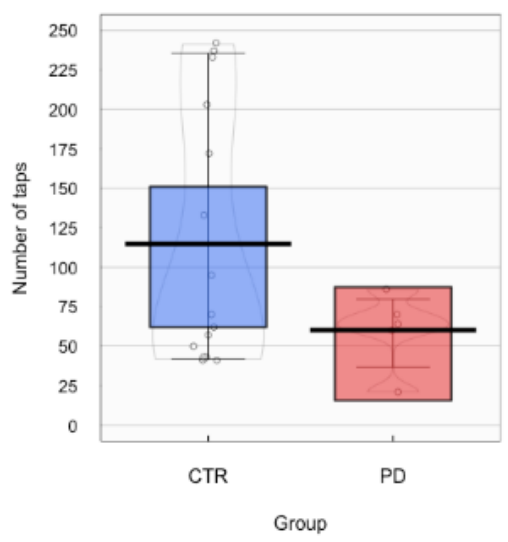

Figure 1. The total number of taps for controls (CTR) compared to people with Parkinson's Disease (PD). Means are shown by black horizontal bars. Individual scores are represented by black circles, slightly jittered for clarity; colored areas represent $95 \%$ highest density intervals, calculated using R's Bayesian Estimation Supersedes the T-Test package, and vertical bars represent the 10th and 90th quantiles. Thin grey lines show the full densities for each group.

The application (Multimedia Appendix, Demonstration Video, and Code Release under the MIT license) was perceived as easy to use and participants could navigate between pages without any issues. They appreciated its simple yet effective design (ratings up to $9 / 10$ and on average, $7 / 10$ for its user interface) and were satisfied with the instructions. 20 participants $(90 \%)$ had never used such an application before. Hence, some participants experienced difficulties in using the smartphone in general. Nobody experienced particular issues regarding the application. 15 participants $(70 \%)$ said that they would use the application again.

However, opinions were mixed about receiving visual feedback from the application. Some participants found warning messages or change in background color distracting. They recommended changing the color of the word TAP within the circles rather than the background. Some participants also found circles too far from each other.

Participants with PD had some hesitation in initiation of movement, which meant that fewer taps occurred at the start of the task than later. Some of them also had difficulties in regulating how softly or hard they tapped, lifting their fingers from the screen after tapping, or moving their fingers between the two circles in between the taps. Finally, their tapping speed typically decelerated, whereas controls tapped steadily.

Regardless of these experiences of having some difficulty with finger tapping, participants with PD perceived the application as an opportunity to contribute to research. One participant with PD was intrigued by the finger-tapping task itself and discussed how their first difficulty experienced before the PD diagnosis was in using a computer mouse. 


\section{Discussion}

Participants in this usability study provided valuable comments and criticisms that informed enhancement of the application in all aspects, especially the user interface. The beta version specifies the two fingers to use for tapping in the instructions in order to reduce confusion and rule out any differences that might result from using different fingers.

Secure login and offline access need to be resolved to protect the privacy of the stored data and enhance its quality. Login can be added to the application to create a profile for the user, which they can securely access. This can be assisted by asking the user to sign a consent form before they begin performing the tasks; allowing researchers to have access to their data. The application currently requires a continuous connection to the Internet to store results in the cloud; without it, the task does not complete successfully. To recover from this error experienced by some participants, in future versions a warning will be displayed to the user and the results stored locally until the connection is re-established, when the data will be uploaded to a server.

Despite its simple and intuitive design, the application still has some significant obstacles. The main limitations are the lack of comparison of data collected by the application to data collected in more controlled clinical settings [18]. The tasks performed without supervision are typically less accurate than clinic-based tests; this is primarily due to users having no guidance on how to perform the tasks $[7,19]$. To improve this, clinical testing of the application needs to be carried out, with the aim of ensuring that the data collected is comparable to clinical assessments. A blinded comparison of supervised and unsupervised data collection sessions will support this.

Future clinical testing will also serve educational purposes, alleviating issues related to resistance and usability problems, which might occur on patients' first-time use of the application. This would reinforce bringing the clinician and patient closer. A means to assist users with answering demographic questions could be to incorporate speech recognition in the application. This would greatly benefit patients, as they then would not have to type responses, which could be difficult for those with tremor symptoms. Looking forward, as finger-tapping tasks are commonly used in neuroimaging studies [20], the context of the application does not need to be limited to PD, but can be applied to quantitative assessment in other neurodegenerative diseases [21,22].

\section{Conclusions}

This user study with 22 participants has indicated that our finger-tapping application provides them with a usable and feasible means for tracking their disease symptoms and progress. Because finger-tapping tasks have been shown to correlate with fine-motor symptoms of PD, they are a useful as a supplementary tool to assist clinicians in the diagnosis and ongoing assessment of PD. The application has the potential to enable convenient and affordable data gathering that may reveal new information about PD to patients and their families, clinicians, and scientists, and to subsequently inform treatment. 


\section{Acknowledgements}

This research has been delivered in partnership with Our Health in Our Hands (OHIOH), a strategic ANU initiative, which aims to transform health care by developing new personalized health technologies and solutions in collaboration with patients, clinicians, and health-care providers. It was conducted when the second author was an ANU Bachelor's student. She received the Paul Thistlewaite Memorial Honours Year Scholarship 2018 from the ANU College of Engineering and Computer Science. We also thank the organizers of the Parkinson's Biomarker Dream Challenge - Sage Bionetworks and the Brains on the Hill event - Australian Brain Alliance. Finally, we wish to extend this acknowledgement to all the participants, advisers, reviewers' who volunteered for the user study and/or provided valuable feedback

\section{References}

[1] Parkinson's Foundation. Statistics 01 (2018).

[2] Lang AE, Lozano AM. Parkinson's disease, N. Engl. J. Med. 339 (1998) 1044-1053.

[3] Torta DM, Castelli L. Reward pathways in Parkinson's disease: Clinical and theoretical implications. Psychiatry Clin. Neurosci. 62 (2008) 203-213.

[4] Pal G, Goetz CG. Assessing bradykinesia in Parkinsonian disorders. Front. Neurol. 4 (2011) 1859-1863.

[5] Rees RN, Acharya AP, Schrag A, Noyce AJ. An early diagnosis is not the same as a timely diagnosis of Parkinson's disease. F1000Res 7 (2018) F1000.

[6] Mathur S, Mursaleen L, Stamford J et al. Challenges of improving patient-centred care in Parkinson's disease. J. Parkinsons. Dis. 7 (2017) 163-174.

[7] Adams WR. High-accuracy detection of early Parkinson's disease using multiple characteristics of finger movement while typing. PLoS One 12(2017) e0188226.

[8] Schrag A. How valid is the clinical diagnosis of Parkinson's disease in the community? J. Neurol. Neurosurg. Psychiatry 73 (2002) 529-534.

[9] Schrag A, Modi S, Hotham S et al. Patient experiences of receiving a diagnosis of Parkin-son's disease. ,J. Neurol. 265 (2018) 1151-1157.

[10] Goetz CG. Unified Parkinson's Disease Rating Scale (UPDRS) and the Movement-Disorder Society sponsored Unified Parkinson's Disease Rating Scale (MDS-UPDRS). Encyclopaedia of Movement Disorders 285 (2010) 307-309.

[11] Lee CY, Kang SJ, Hong SK et al. A validation study of a smartphone-based finger tapping application for quantitative assessment of bradykinesia in Parkinson's disease. PloS one 11(2016) e0158852.

[12] Thorp JE, Adamczyk PG, Ploeg HL, Pickett KA. Monitoring motor symptoms during activities of daily living in individuals with Parkinson's disease. Front Neurol. 9 (2018):1036.

[13] Cosgrove J, Lacy S, Jamieson S et al. Finger tapping and cognition in Parkinson's. J. Neurol. Neuro-surg. Psychiatry 86 (2015) e4.

[14] Friedman B, Kahn PH, Borning A et al. Value sensitive design and information systems. Philosophy of Engineering and Technology 16 (2013) 55-95.

[15] Razzouk R, Shute V. What is design thinking and why is it important? Review of Educational Research 210 (2012) 33

[16] Kohavi R, Longbotham R. Online controlled experiments and a/b testing. Encyclopedia of Machine Learning and Data Mining, Springer, Boston, MA, USA, 2017, 922-929.

[17] Henze N, Boll S. It does not fitts my data! Analysing large amounts of mobile touch data. Lecture Notes in Computer Science 6949 (2011) 564-567.

[18] Ingram LA, Carroll VK, Butler AA et al. Quantifying upper limb motor impairment in people with Parkinson's disease: a physiological profiling approach. PeerJ, 9 (2021) e10735.

[19] Lan BL, Yeo JH. Comparison of computer-key-hold-time and alternating-finger-tapping tests for earlystage Parkinson's disease. PLoS One 14 (2019) e0219114. 
[20] Witt ST, Laird AR, Meyerand ME. Functional neuroimaging correlates of finger-tapping task variations: An ALE meta-analysis. NeuroImage 42 (2008) 343-356.

[21] Bechtel RI, Scahill HD, Rosas et al. Tapping linked to function and structure in premanifest and symptomatic Huntington disease. Neurology 75 (2010) 2150-2160.

[22] Lamers I, Kelchtermans S, Baert I et al. Upper limb assessment in multiple sclerosis: A systematic review of outcome measures and their psychometric properties. Arch. Phys. Med. Rehabil. 95 (2014)1184-200. 